\title{
REFERENCES
}

1. R. L. Moore, Concerning triods in the plane and the junction points of plane continua, Proc. Nat. Acad. Sci. U.S.A. vol. 14 (1928) pp. 85-88.

2. - Foundations of point set theory, Amer. Math. Soc. Colloquium Publications, vol. 13, 1932.

3. R. L. Moore and J. R. Kline, On the most general plane closed point set through which it is possible to pass a simple continuous arc, Ann. of Math. vol. 20 (1919) pp. 218-223.

4. R. L. Moore, Concerning upper semi-continuous collections of continua, Trans. Amer. Math. Soc. vol. 27 (1925) pp. 416-428.

5. G. S. Young, Interior and border transformations on surfaces, Bull. Amer. Math. Soc. Abstract 52-5-220.

6. Herman Cohen, Some results concerning homogeneous plane continua, Duke Math. J. vol. 18 (1951) pp. 467-474.

The Institute for Advanced Study and

The University of Pennsylvania

\section{DIMENSION AND DISCONNECTION}

\section{BYRON H. MCCANDLESS}

Let $X$ be a semi-compact separable metric space. We shall prove the following theorem using results found in Hurewicz and Wallman's book Dimension theory (Princeton University Press, 1948):

THEOREM. $\operatorname{dim} X \leqq n$ if and only if any closed subset of $X$ containing at least two points can be disconnected by a closed set of dimension $\leqq n-1$.

The necessary and sufficient condition stated in the theorem was found in looking for an $n$-dimensional analogue of the property of a space being totally disconnected (property $\alpha_{0}$ below) and will be denoted by $\alpha_{n}$.

Hurewicz and Wallman show (p. 20) that the following three properties of the space $X$ are equivalent:

$\alpha_{0} . X$ is totally disconnected.

$\beta_{0}$. Any two points in $X$ can be separated.

$\gamma_{0}$. Any point can be separated from a closed set not containing it, that is, $\operatorname{dim} X=0$.

They also show (p. 36) that the following $n$-dimensional analogues of $\beta_{0}$ and $\gamma_{0}$ are equivalent:

$\beta_{n}$. Any two points in $X$ can be separated by a closed set of dimension $\leqq n-1$.

Received by the editors December 5, 1951. 
$\gamma_{n}$. Any point can be separated from a closed set not containing it by a closed set of dimension $\leqq n-1$, that is, $\operatorname{dim} X \leqq n$.

As we have already noted, the $n$-dimensional analogue of $\alpha_{0}$ is: $\alpha_{n}$. Any closed subset of $X$ containing at least two points can be disconnected by a closed set of dimension $\leqq n-1$.

Obviously $\gamma_{n}$ implies $\beta_{n}$ and $\beta_{n}$ implies $\alpha_{n}$. We shall show that $\alpha_{n}$ implies $\gamma_{n}$. It will then follow, in analogy with the 0 -dimensional case, that $\alpha_{n}, \beta_{n}$, and $\gamma_{n}$ are equivalent, thus proving the theorem. The known equivalence of $\beta_{n}$ and $\gamma_{n}$ is not used in our proof.

We are to show that if the space $X$ possesses property $\alpha_{n}$, then $\operatorname{dim} X \leqq n$. Since $X$ is the countable union of compact sets we need only to show, by virtue of the sum theorem for dimension $n$ (p. 30), that this is true of a compact space. Therefore, from this point on, let $X$ denote a compact separable metric space. The method of the following proof is due essentially to Hurewicz and Wallman. Let $C$ be a closed subset of $X$ and $f$ a mapping of $C$ in the $n$-sphere $S_{n}$; it suffices to show (p. 83) that $f$ can be extended over $X$. Suppose, to the contrary, that $f$ cannot be extended over $X$. There then exists (p. 94) a closed set $K$ such that:

(1) $f$ cannot be extended over $C \cup K$, but

(2) $f$ can be extended over $C \cup K^{\prime}$ where $K^{\prime}$ is any proper closed subset of $K$.

(This statement is false for spaces which are only locally compact.) If $K$ contains at most one point, $f$ clearly can be extended over $C \cup K$ in contradiction to (1). Therefore $K$ contains at least two points. It then follows from property $\alpha_{n}$ that there exist (p. 47) two proper closed subsets $K_{1}$ and $K_{2}$ of $K$ such that $K_{1} \cup K_{2}=K$ and $\operatorname{dim} K_{1} \cap K_{2}$ $\leqq n-1$. By (2), $f$ can be extended to mappings $f_{1}$ and $f_{2}$ over $C \cup K_{1}$ and $C \cup K_{2}$ respectively. Since $\operatorname{dim} K_{1} \cap K_{2} \leqq n-1$, each of the extensions $f_{1}$ and $f_{2}$ can be extended (p. 88) over the union $C \cup K$ of $C \cup K_{1}$ and $C \cup K_{2}$. Therefore $f$ can be extended over $C \cup K$ in contradiction to (1). This contradiction proves that $f$ can, in fact, be extended over $X$. Consequently $\operatorname{dim} X \leqq n$, as was to be proved. 